\title{
Design Considerations of Contemporary Ceramic Art Exhibits
}

\section{Huang Zhang}

Art and Design School, Wuhan University of Technology, Wuhan, China

\section{Email address:}

664782154@qq.com

\section{To cite this article:}

Huang Zhang. Design Considerations of Contemporary Ceramic Art Exhibits. Science Innovation. Vol. 5, No. 5, 2017 , pp. $298-302$. doi: 10.11648/j.si.20170505.20

Received: June 23, 2017; Accepted: July 20, 2017; Published: August 7, 2017

\begin{abstract}
In modern society, we should not only inherit the tradition, but also transform it with innovative thinking. Most of China's ceramic art enthusiasts still stay on the traditional view of ceramic design. However, this kind of cling to the traditional thought cannot adapt to the contemporary art environment. This paper studies the design method of contemporary ceramic art exhibits with the theory of modern design. This paper analyzes the design context of contemporary ceramic art exhibits from the angle of psychology, studies the manifestation of contemporary ceramic art exhibits in the modern context, tries to return to nature, and finds its own concise design form and unique design language in naturalism.
\end{abstract}

Keywords: Ceramic Art Exhibits, Modern Design Method, Naturalism, Simple Design

\section{当代陶瓷艺术陈列品的设计思考}

\section{张璜}

艺术与设计学院, 武汉理工大学, 武汉, 中国

邮箱

664782154@qq.com

摘要：现代社会, 对于传统不仅要传承, 同时也要以创新的思维对其进行改造。国内大多数陶瓷艺术爱好者仍仅停留 在传统的层面看待陶瓷设计。然而这种固守传统的思想无法适应当代的艺术环境。文章以现代设计的理论方法去研究 当代陶瓷艺术陈列品的设计方法; 从心理学的角度去分析当代陶瓷艺术陈列品的设计语境; 研究当代陶瓷艺术陈列品 在现代语境下的表现方式，力图做到回归自然，在自然主义中找到自己简洁的设计形式与独特的设计语言。

关键词: 陶瓷艺术陈列品, 现代设计方法, 自然主义, 简约设计

\section{1. 引言}

经济的发展带来了工业的进步, 现代社会人们生活节 奏也变快, 而形形色色的产品也开始充斥在我们的生活空 间中，使原本繁忙的生活显得更加拥挤。陶瓷艺术也进入 这一行列, 大多数陈列品或艺术作品出现在室内空间中。 而室内空间是重要的生活场所, 所以室内环境对于生活品 质有极大的调节作用。因此, 陶瓷艺术陈列品进入我们生
活的空间, 也意味着在当代社会语境下对陶瓷艺术陈列品 要提出新的设计要求。 


\section{2. 当代陶瓷艺术陈列品设计现状}

\section{1. 现代主义下陶瓷艺术陈列品的选择}

审美是人类自我意识的一种表现, 从历史发展的角度 来看, 社会存在决定社会意识, 现代社会生活方式促成了 新的艺术风尚。在后现代艺术思潮的引领下, 设计和审美 都更加倾向一种前卫的思想, 更注重一种纯粹生活的理念。 图1八木一夫的《萨姆萨先生的散步》, 将花瓶所有插口 都封起来, 在陶瓷艺术界引起震动, 其后很多设计师也开 始了向纯粹的具有前卫风格的造型设计转变。这种摆脱对 传统陶瓷设计的束缚, 极具设计感的现代主义陶瓷艺术品 的设计形式在现代生活中也越来越受人们的青睐。

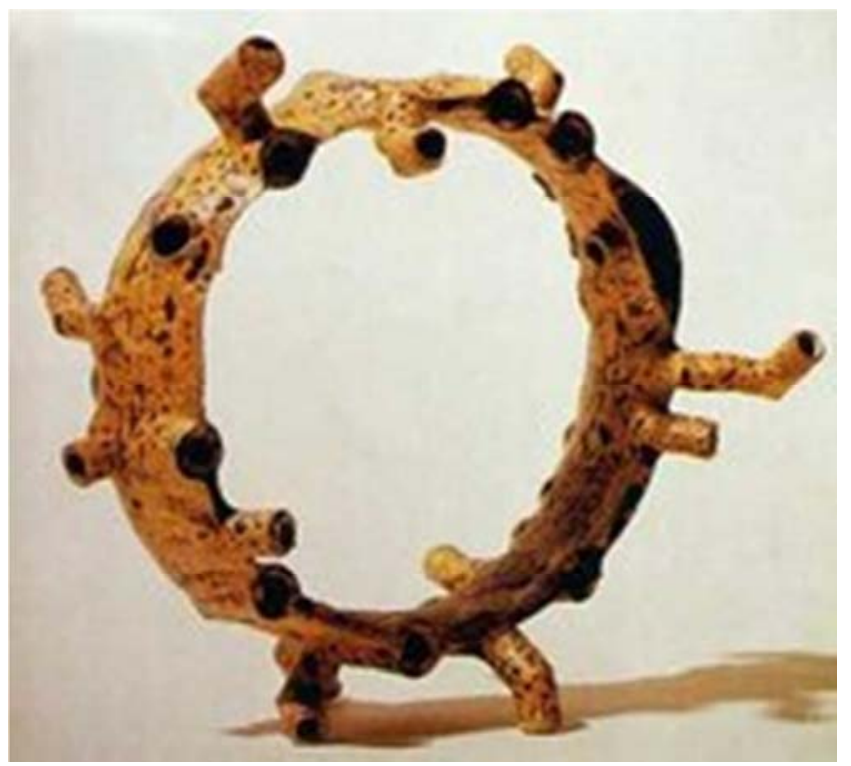

图1 八木一夫《萨姆萨先生的散步》。

\section{2. 现代审美语境下陶瓷艺术陈列品的问题}

当代的陶瓷艺术陈列品同样也存在着很多的局限性, 首先, 大多陶瓷艺术品制作工艺精良, 但缺乏创新性, 与 现代主义风格格格不入。精美的陶瓷艺术被赋予的更多是 一种繁复的工序、精细的描图和富丽的色彩。但现代审美 并不是一味的追求繁杂、富贵与浮华, 是倾向于简约自然 的风尚, 所以, 那些工艺精良的陶瓷艺术品在现代生活中 运用范围十分局限。其次, 很多陶瓷匠人思想守旧, 在现 代生活中运用性不强。出于对老一辈陶瓷艺人的尊重, 传 统的一些陶瓷艺术品被誉有很高的艺术价值。当代很多艺 人们仍延续他们的风格, 包括现有的一些陶瓷研究所, 设 计和生产的陶瓷仍然是十分传统的风格, 装饰图案上仍采 用极为传统的山水人物等, 器形创作上也未见突破。显然 这种传统的创作方式无法与现代生活接轨。如图2, 国内 某陶瓷展厅陈列的陶瓷艺术品, 作品无论从工艺技法还是 画工上都十分精湛, 但这些精美的陶瓷艺术品与现代生活 审美理念相差甚远。

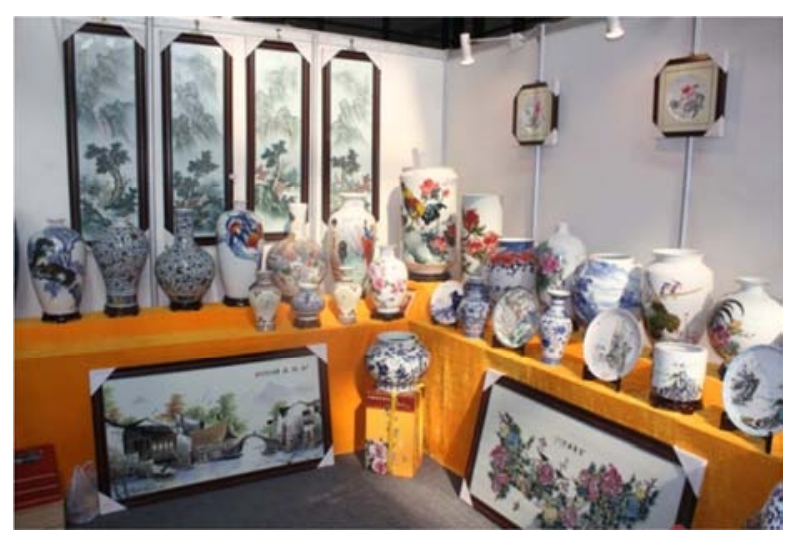

图2 作者自摄。

所以, 如何继承传统, 必须以创新的思维对待。现代 陶艺设计不仅是技艺的表达更是对生活的一种诠释, 正如 张道一先生所说“现代陶艺是一种社会意识形态，通过塑 造形象具体地反映社会生活, 表现作者的思想感情和政治 倾向的艺术”。那么, 陶瓷艺术陈列品要与现代生活相融, 就要在现代主义风格倡导下所形成的审美方式中寻找传 统陶瓷工艺的创新之道。

\section{3. 现代空间中陶瓷艺术陈列品的审美特征}

艺术源于生活, 现代社会生活水平提高, 生活质量的 要求也越来越高, 审美心理发生了巨大的变化, 传统的陶 瓷艺术已经无法适应现代物质与精神的生活方式, 现代陶 艺作品经常作为一种陈设品出现在空间环境中。现代空间 追求简洁而明朗的设计, 通过空间、材料、颜色、比例, 来达到简洁自然的目的。在这种空间环境中, 陶瓷艺术陈 列品也必须要形成适合现代环境的审美特征。

\section{1. 陶瓷艺术陈列品的审美体验}

格式塔心理学认为, 人之所以对某个事物产生美感, 主要是审美主体与事物有着某种相似之处, 当两个相似的 主客体相遇, 两者便产生所谓心理的共鸣, 唤起主体审美 的情感力量 ${ }^{1}$ 。在空间环境中，当整个空间环境布局与心 境达到一致的时候, 自然就会感到平静、舒适，同时达到 人们心理对于秩序与和谐的追求, 美感也由此产生。现代 主义空间环境追求舒适自然的生活方式, 21 世纪著名建筑 家隈研吾 (Kengo Kuma) 提出的 “负建筑” 理念就试图 将建筑与周围的环境融合, 将建筑与人、自然进行交融与 延伸 ${ }^{2}$ 。陶瓷艺术品作为空间装饰之一, 也要符合现代空 间审美的理念和趋势, 在现代审美视角下, 与生活空间相 契合。图3, 日本著名设计师黑川雅之 (Masayuki Kurokawa) 设计的一组餐具套装强调简洁, 产品的形状极度精简, 纯 粹的白色, 以及大小餐盘叠加形成的秩序与韵律的美感, 都像是生命的节奏。因此, 这组作品不仅具有餐具的实用 功能, 也以空间的视角去审视作品, 触发人们对生活的哲 理思考。

1 朱光潜著. 《西方美学史》 $[\mathrm{M}]$. 北京: 人民文学出版社, 2002.5

2 隈研吾著. 《新建筑入门》[M]. 中信出版社,2011.1 

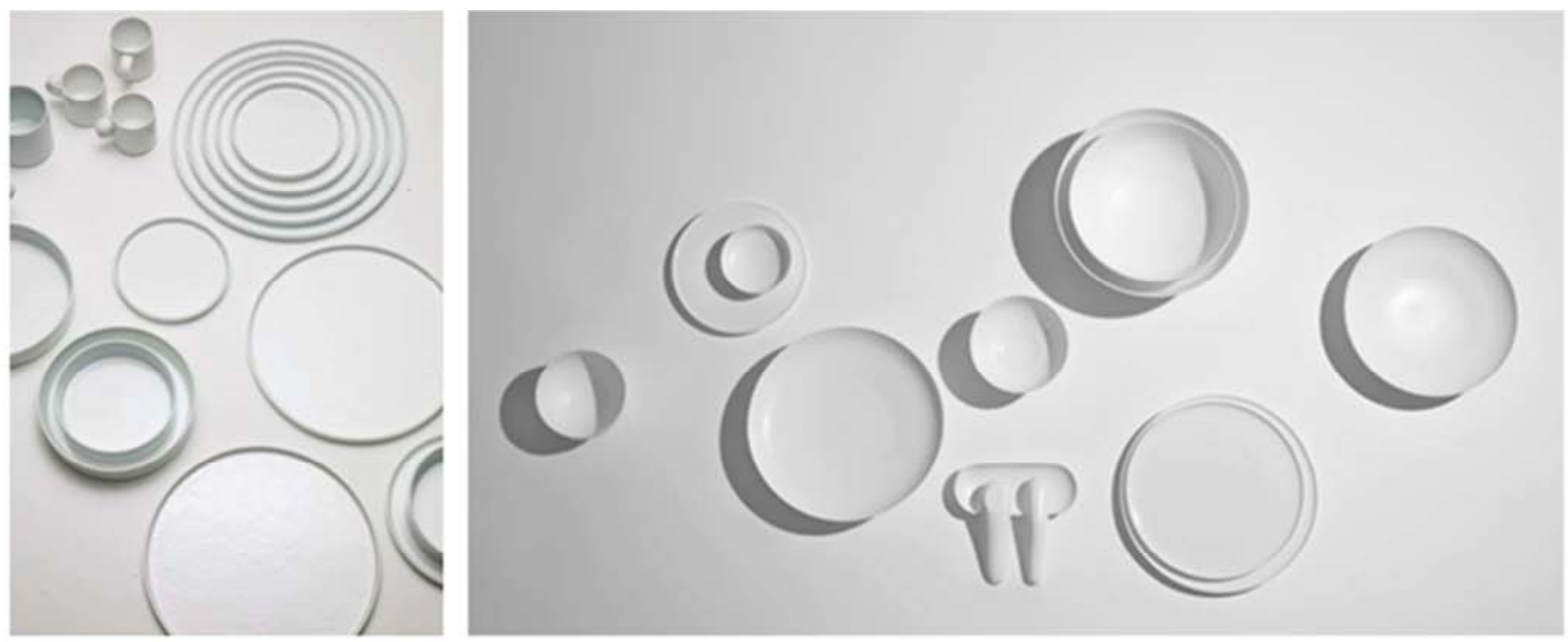

图3 黑川雅之《ZK餐具套装》。

\section{2. 陶瓷艺术陈列品审美的系统性与整体性}

\subsection{1. 统一的风格形式}

从形式美感的角度来看, 陶瓷艺术陈列品被置放在 某一处空间时, 同样是受视觉支配所形成的艺术形式, 因此, 陶瓷艺术陈列品在空间中的审美同样遵循着变化 与统一的形式美法则。在室内建筑作品中, 丰富的元素 使得设计手法与表现形式多样。但是, 变化中追求统一, 统一于一个符合空间场景的视觉形象, 这样才能形成一 定的空间视觉秩序和形式感。而陶瓷艺术陈列品作为存 在于空间范围内的一种艺术形式, 自然要与周围的空间 保持一致风格。

\subsection{2. 合适的空间比例}

陶瓷艺术陈列品被越来越多的人作为一种室内的装 饰而存在, 而这种存在必然遵循一定的秩序法则。毕达哥 拉斯学派讲究数是万物的本原, 万物按照一定的数量比例 而构成和谐的秩序，比例被认为是基本的形式美原则之一, 广泛的运用在设计创作之中 ${ }^{3}$ 。现代主义设计中同样遵循 这一原则, 现代空间关系中的审美要把握好陈设品体量与 空间的关系，陈设的陶艺品要从居室的面积、层高，来把 握好其体量和空间感。在视觉心理作用下，大尺度空间配 以大尺度的陈设品, 反之亦然。要做到在空间中寻找合适 的体量, 就要考虑陶瓷作品本身的造型度量的大小在空间 环境中的比例。

\section{4. 当代陶瓷艺术陈列品的新形式与新语言}

\section{1. 自然主义下陶瓷艺术陈列品的新形式}

庄子说“天地有大美而不言”，东方自然主义风格倡 导“回归自然”, 美学上推崇自然, 结合自然。中国关于 自然的美学思想十分丰富, 从老子的“道法自然”到《园

3 朱光潜著. 《西方美学史》 [M]. 北京: 人民文学出版社, 2002.5
冶》中的“虽由人作，宛自天开”，都表达出对自然的崇 尚。在今天高速发展的社会生活中, 道法自然的思想不 断平衡人们的物质与精神需求。然而在现代主义的引导 下, 陶瓷艺术陈列品必然也要找到属于自己独特语言的 自然主义风格。

\subsection{1. 简约的设计风格}

在老子的美学思想中, 有一种重要的美学观一一朴素 自然美。“见素抱朴, 少思寡欲, 绝学无忧”, 朴, 就是简 单、质朴、不加雕饰; 素, 就是本色、本真、素约, 而自 然主义中也正是这种简朴和单纯来净化人们的心灵。日本 设计师原研哉（Kenya Hara）曾说过“简单最好”, 它所主 张的生活方式和追求的装饰正属于简的美学范畴 5 。现代 主义同样提倡简约来主导设计风格, 著名产品设计师深泽 直人 (Naoto Fukasawa) 在设计上追求极致简洁, 寻求设 计与心灵的对话, 在现代审美疲劳的时代, 倾听人们内心 的声音, 在避免给精神增加负担的同时发现内心深处的需 求, 于是化繁为简, 就像一个自然调整的过程。如图4, 他为“ \pm 0 ”品牌设计的加湿器, 产品在形式上极度简洁, 简 化为最基础的圆形, 附着类似陶瓷的材质, 这种极简设计 带给人诗意般的宁静。

陶瓷设计与产品设计如出一辙, 在这种设计风格倡导 下产生的陶瓷艺术品, 也讲究简约的艺术。首先形式的简 约直接明了的传达设计理念。现代造型语言中, 简约是浪 漫与时尚的碰撞, 在满足功能的基础上, 用凝练的设计语 言直抒心怀; 其次通过造型的节奏、韵律、动感、层级和 体量来获得简洁、雅致的审美体验; 同样, 清新素雅的色 彩语言也能传达出陶瓷设计的简约之意, 体现出现代人的 审美格调与品位。

4 老子. 《道德经》第十九章

5 [日]原研哉著. 张钰译. 欲望的教育 [M]. 桂林: 广西师范大学出版社, 2012.3 


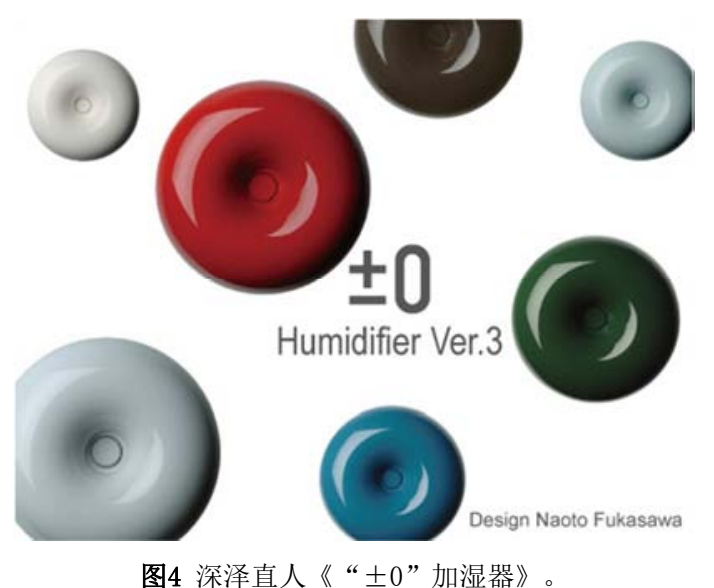

4.1.2. 秩序的形态要求

秩序无处不在, 自然中万物都是遵循一定秩序而生长, 我们也生活在秩序中, 感受着秩序带给我们心理上的安定, 同时也将杂乱的事物向着有序的方向调整。对于艺术作品 中的秩序，狄德罗说：“艺术产品中有本质美、人类创造 美和体系美: 本质美在于秩序; 人类创造美在于艺术家依 赖而又灵活地运用法则, 或者说得清楚一点, 就是在于对 某种秩序的选择; 体系美则产生于观察 ${ }^{6}$ ”。对于陶瓷艺术 品的创作同样需要对秩序有所考究。无论是造型的对称, 色彩的融合, 还是图案的重复等等, 通过平行构成或是几 何构成的手法将元素排列组合, 当他们被秩序的组合在一 起是, 才能构成最完美的形态。图5, 当代艺术家艾未未 创作的《一亿颗陶瓷瓜子》, 每一粒葵花籽作为不同的个 体单独存在, 当一亿颗瓜子铺满整个空间, 个体瞬间融合, 形成空前的秩序与统一。虽然是一件大型装饰作品, 但艺 术家对于个体形态与秩序关系的理解, 让我们对于秩序本 身有了新的认识。

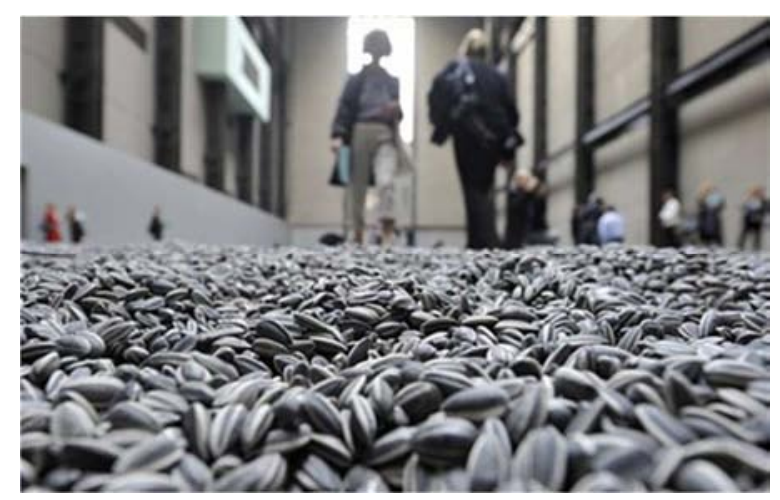

图 5 艾未未《一亿颗陶瓷瓜子》。

\section{1. 3. 韵律的美感塑造}

宗白华先生在谈到中国绘画时指出: “它所描写的对 象、山川、人物、花鸟、虫鱼, 都充满着生命的动一气 韵生动 ${ }^{7}$ ”。人们从画中感受到生命的跳动与活跃, 正是这 种生命的韵律使美感油然而生。同时, 韵律美与毕达哥拉

6 《狄德罗论美文选》 [M]. 北京: 人民文学出版社, 1984.16 7 宗白华:《美学与意境》,人民出版杜1987年版,第9、10页
斯学派所倡导的比例美、和谐美异曲同工。在陶瓷艺术品 设计中, 无论是造型、比例, 还是色彩、肌理, 都应该以 最和谐的方式运用在设计之中, 只有这样陶瓷艺术品的创 作才能带给我们生机与活力, 感受到韵律之美。如图6, 当代英国设计师马修·钱伯斯（Matthew Chamber）设计的 同心层状陶瓷雕塑, 将抽象复杂的立体形态与陶瓷相结合, 使用最原始的陶土, 有如自然般的土石质感, 层层叠叠之 中透显出现代陶瓷艺术的美感, 在自然、简洁主义的刻化 下不自觉的展现出的生命的律动。

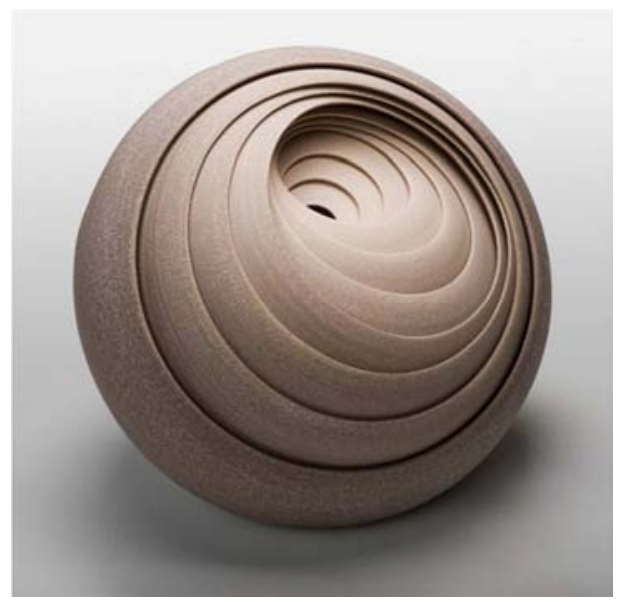

图 6 马修・钱伯斯同心层陶瓷雕塑。

\section{2. 现代设计中陶瓷艺术陈列品的新语言}

\subsection{1. 材料的天然特质}

日本著名民艺学家柳宗悦（Sooetsu Yanagi）在《工 艺文化》一书中说道: “材料是其天籁, 其中浓缩了很多 人工智慧难以预料的神秘。”所谓“材美工巧”，材料的选 择直接关系到器物的功能和审美。设计师善于从大自然中 汲取灵感, 利用天然资源的价值, 在设计中尽情释放原材 料的鬼力。图7, 伦敦智利籍设计师利维亚 -马林 (Livia Marin）创作的一组陶瓷作品《Broken Things》打破了人 们对陶瓷材料的固化观念, 以陶瓷材质塑造逼真的融化效 果, 艺术家融化破碎的陶瓷, 结果陶瓷融化后保有原来的 纹饰, 仿佛液体从瓷器中流出, 似乎诉说生活中点点滴滴 的记忆, 象征一种永不流逝的力量。

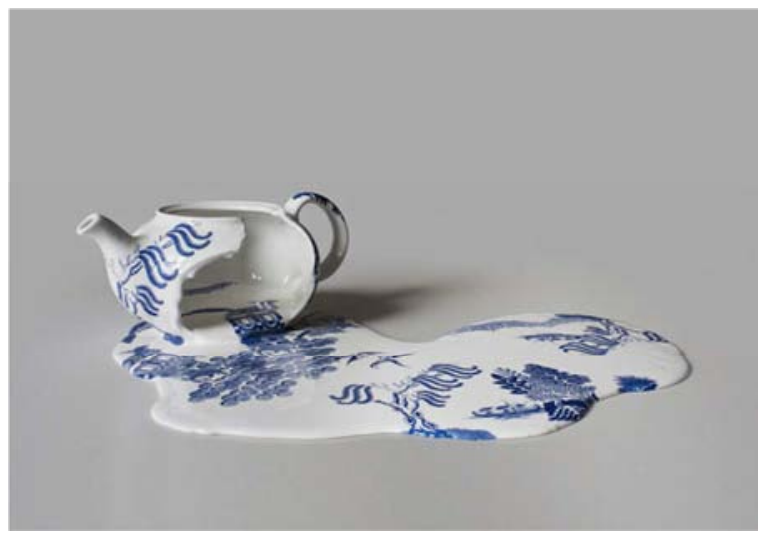

图 7 利维亚・马林《Broken Things》。 


\subsection{2. 器型的独特语言}

传统的陶瓷设计造型多以盘、罐、瓶等较常规的器皿 造型, 具有端庄、规整、对称的古典美，但在当代国际艺 术环境下, 这样传统的陶瓷设计似乎并未有所创新。在自 然主义设计风潮的指导下, 陶瓷器型在自然中汲取灵感, 进行设计创作。如图8, 西班牙艺术家阿尔伯托·巴斯托斯
（Alberto Bustos）设计的陶艺作品, 受植物形态的启发, 用陶瓷艺术塑造自然之美。同时设计打破常规造型思维, 设计巧妙而独特, 用陶瓷塑造出纸艺作品之态, 有着当代 设计的视野, 追求设计语义的纯粹性与独特性, 利用造型 诠释万物生长之意。

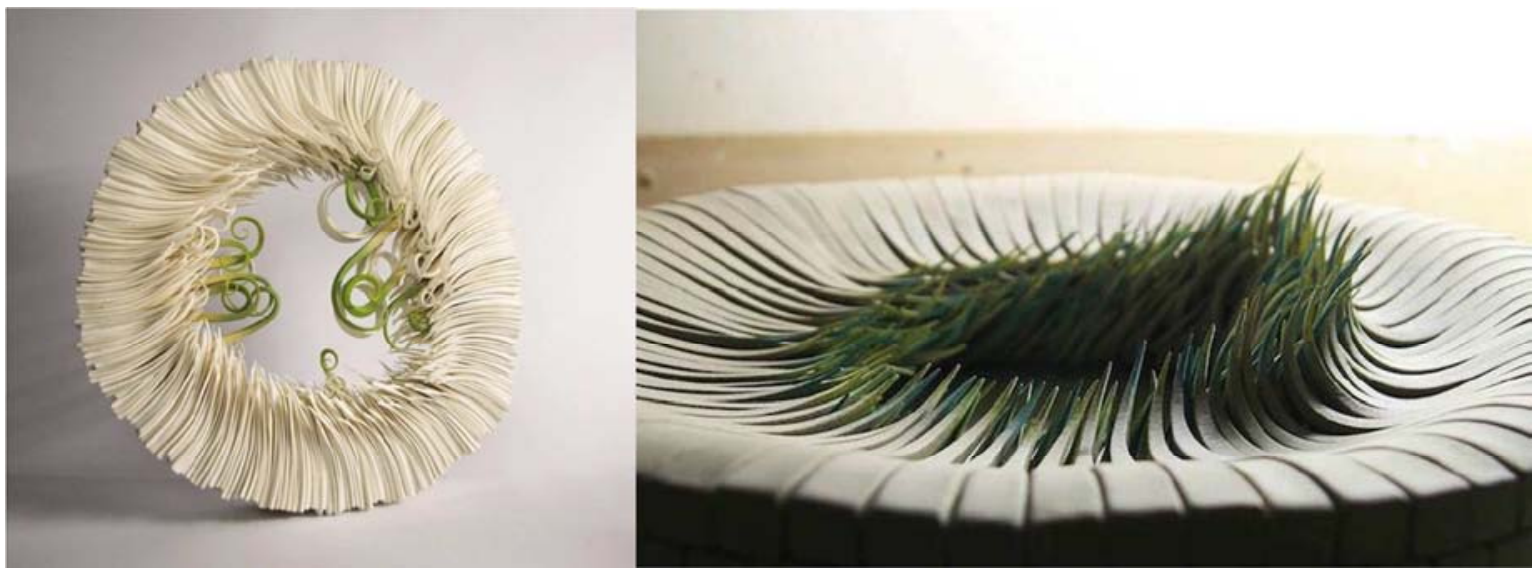

图 8 阿尔伯托 -巴斯托斯陶艺作品。

\section{5. 结论}

陶瓷艺术作为一门独具特色的艺术门类, 有着自己深 刻的艺术独特性。但在面对现代化的高速发展与当代的社 会生活环境时, 当代陶瓷艺术陈列品应该具有更广阔的延 展性, 而不是一味的固守传统, 应该在现代主义对自然的 崇尚、对简约主义的追求中, 突出陶瓷艺术品对自然、对 生活的思考, 在自然主义形态中吸收有益元素。通过对节 奏、韵律、秩序关系的重新整理与设计, 融入当下空间语 境中, 找到当代陶瓷艺术陈列品的设计形式, 将陶瓷材质 发挥到极致, 从器型上突破, 打破空间局限, 富于陶瓷艺 术时代性。因此，作为当代的陶瓷设计者，致力于将空间 环境、自然形态语言与现代陶瓷艺术完美结合, 找到当代 艺术中陶瓷艺术的自我表达方式, 这既是一种挑战也是我 们肩上不可推卸的责任。

\section{参考文献}

[1] 朱光潜著. 《西方美学史》 $[\mathrm{M}]$. 北京: 人民文学出版社, 2002.5。

[2] (美)卡罗尔·D. 巴格著. 国外家居设计精选-典雅室内设计 $[\mathrm{M}]$. 北京：中国建筑工业出版社，2005。

[3] [日]隈研吾著. 《新建筑入门》 $[\mathrm{M}]$. 北京: 中信出版社, 2011.1。

[4] [日]原研哉著（张钫, 译）欲望的教育[M]. 广西: 广西师 范大学出版社, 2012。
[5] 老子. 《道德经》 $[\mathrm{M}]$. 第十九章。

[6] 《狄德罗论美文选》[M]. 北京: 人民文学出版社, 1984.16。

[7] 宗白华著. 《美学与意境》 [M]. 北京: 人民出版杜, 2009 年版。

[8] 李文嘉. 从物性到诗性一禅意美学下的陶瓷产品创新设计 研究 $[J]$. 中国陶瓷. 2016.1。

[9] 郑玉梅. 极简主义风格在生活陶艺设计中的应用研究[J]. 中 国陶瓷.2016.4。

[10] 陈潇玮. 还物以本真一卢志荣自然主义家具设计解析 [J]. 装 饰. 2012.5。

[11] 郭天宝. 基于空间优化的居室陈列品设计研究[D]. 吉林大 学, 2015.4。

\section{作者简介}

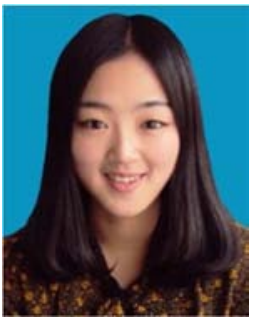

张璜, 1993年7月, 女, 汉, 江西景 德镇

武汉理工大学设计学博士 